\title{
Optimal pricing for internet service providers: Fuzzy geometric programming model
}

\author{
S. J. Sadjadi ${ }^{1 *}$, A. Yousefli ${ }^{1}$ and R. Ghezelsoflou ${ }^{2}$ \\ ${ }^{1}$ Department of Industrial Engineering, Iran University of Science and Technology, Narmak, Tehran, Iran. \\ ${ }^{2}$ Department of Management, Islamic Azad University, South branch, Tehran, Iran.
}

Accepted 8 February, 2011

\begin{abstract}
An internet service provider (ISPs) normally purchases a bandwidth from a vendor and allocates it to different services. The primary concern is to maintain a quality service and, at the same time, maximize the profitability. This paper presents a mathematical model to find the optimal pricing strategy for an internet service provider when the primary concern on many pricing strategies is the absence of historical data for services which makes it difficult to provide a good estimate on the necessary pricing information such as price elasticity and the parameters are taken as a triangular fuzzy numbers. This paper presents a mathematical model to find the optimal pricing strategy in the fuzzy environment. The resulted problem is formulated as a general form of possibilistic geometric programming (PGP). The implementation of the proposed method is demonstrated via a case study on an ISP in Iran.
\end{abstract}

Key words: Revenue management, pricing, geometric programming, fuzzy programming, internet service provider.

\section{INTRODUCTION}

Revenue Management (RM) has considerable potential for economical production in order to maximize the total profit. It can significantly help organizations keep their advantages in competitive markets. Belobaba, (1987), McGill and Ryzin (1999) discussed about the origin of the revenue management. McGill and Ryzin (1999) categorized revenue management problems into forecasting, overbooking, pricing and capacity control. Chiang et al. (2007) extended their model by considering additional assumptions. As McGill and Ryzin (1999) mentioned, pricing is one of the most important aspects of RM. The optimal pricing has been investigated by many researchers during the past few decades. In the proposed models, some factors such as selling price, customer segmentation, marketing expenditure, etc have been frequently considered as the most effective factors in the optimal pricing problem. Burger and Fuchs (2005) argued about the effects of dynamic pricing on RM and showed

${ }^{\star}$ Corresponding author. E-mail: sjsadjadi@iust.ac.ir. Tel: 0982177240129 . Fax: 0982177240482. that it can lead to a decrease in complexity of pricing process. Frank (2003) discussed about application of sixsigma at pricing. Lee and Kim (1993) are the first people who proposed an integrated marketing and production models. They considered demand as an exponential function of price and marketing expenditures and, at the same time, the cost of production was also considered as a function of demand. Therefore, the resulted problem was formulated as a geometric programming model. In another attempt, Kim and Lee (1998) extended their model when the capacity of a market was also incorporated either fixed or variable. Sadjadi et al. (2005) developed Lee's model under some additional assumptions. In other research, Sadjadi et al. (2010) presented a new marketing and pricing model in the fully uncertain environment where decision variables were fuzzy numbers. Also, they developed a decision support system to inference vector of decision variables without solving geometric programming problem directly. Tsaia and Hung (2007) proposed an integrated real options (IRO) approach for solving competitive/dynamic pricing and RM problem in internet retailing where environment was uncertain. They used analytic hierarchy process (AHP) to 
determine the weights of the auction commodities. Nair and Bapna (2001) studied the internet service provider (ISP) market structure and explained that demand and supply in this sector is different from other industry sectors, so that demand and service happened at the same time. In other words, the clients, at any time of day, may require the services and therefore, every moment system must be ready to serve and the possibility of overbooking does not exist.

Note that we have a high level of competition on ISP market on one side and technology is under significant changes on a daily basis and there is a need to develop a good strategy for assigning the suitable price for ISP services.

In this paper, a mathematical model is developed for ADSL service pricing. Since most of the real world problems occurred in the uncertain environment, to tackle this uncertainty, the proposed model is formulated in the fuzzy environment as possibilitic geometric programming model. To show the performance of the presented model, details of implementation of proposed method on one of ISP's located in Iran will be explained.

\section{PROBLEM STATEMENT}

A fuzzy pricing model for ISP services is developed under the following assumptions and notations:

\section{Notations}

$\alpha_{j}$ : Selling price elasticity to demand

$Y_{\mathrm{j}}$ : Type of service ( $\mathrm{kb} /$ second)

$\beta_{j}:$ Share ratio

$\mathrm{P}_{\mathrm{j}}$ : Selling price (decision variable)

$\mathrm{D}_{\mathrm{j}}$ : Services demand

B: Total bandwidth that company receives per period $k_{j}$ : Predetermined constant

\section{Assumptions}

I: Selling price elasticity to demand is uncertain and depicted in the form of triangular fuzzy number $\tilde{\alpha}=\left(\alpha_{l}, \alpha_{m}, \alpha_{r}\right)$.

II: $\tilde{k}$ is uncertain and depicted in the form of triangular fuzzy number $\tilde{k}=\left(k_{l}, k_{m}, k_{r}\right)$

III: Price is a function of demand so that it has inverse relation with the selling price as follows:

$$
D=\tilde{k} P^{-\tilde{\alpha}}
$$

Based on the notations and assumptions expressed, the pricing for ISP services is developed in fuzzy environment.

\section{Fuzzy pricing model}

In this section, the pricing model for ISP services is developed in the fuzzy environment. As mentioned before, the objective function is the maximization of revenue and there is a constraint on bandwidth. If there are in different services, mathematical pricing model can be formulated as follow,

$\max \sum_{j=1}^{n} P_{j} D_{j}$

subject to

$\sum_{j=1}^{n} \frac{\gamma_{j} D_{j}}{\beta_{j}} \leq B$

Replacing D in Model (2) by (1) yields:

$\max \sum_{j=1}^{n} \tilde{k}_{j} P_{j}^{1-\tilde{\alpha}_{j}}$

subject to

$\sum_{j=1}^{n} \frac{k_{j} \gamma_{j} P_{j}^{-\widetilde{\alpha}_{j}}}{\beta_{j}} \leq B$

Model (3) is a possibilitic geometric programming that $\hat{\alpha}$ and $\hat{k}$ have triangular possibility distribution. In order to

solve Model (3), fuzzy goal programming method is used. Following goal programming concept, an aspiration level such as $Z=\left(Z_{l}, Z_{m}, Z_{r}\right)$ is chosen and the objective function is added to constraint via $\tilde{Z}$ as follows:

$\max \tilde{Z}$

subject to

$\sum_{j=1}^{n} \tilde{k}_{j} P_{j}^{1-\tilde{\alpha}_{j}} \geq\left(Z_{l}, Z_{m}, Z_{r}\right)$

$\sum_{j=1}^{n} \frac{\tilde{k}_{j} \gamma_{j} P_{j}^{-\tilde{\alpha}_{j}}}{\beta_{j}} \leq B$

Model (4) is a possibilitic geometric programming in which $\widetilde{\alpha}_{j}$ and $\tilde{\mathrm{k}}_{\mathrm{j}}$ have triangular possibility distributions. 
After defuzzification, Model (4) is reformulated as Model (5):

$\max \sqrt[3]{Z_{l} \cdot Z_{m} \cdot Z_{r}}$

subject to

$\sum_{j=1}^{n} k_{j}^{l} P_{j}^{\left(1-\alpha_{j}^{r}\right)} \geq Z_{l}$

$\sum_{j=1}^{n} k_{j}^{m} P_{j}^{\left(1-\alpha_{j}^{m}\right)} \geq Z_{m}$

$\sum_{j=1}^{n} k_{j}^{r} P_{j}^{\left(1-\alpha_{j}^{r}\right)} \geq Z_{r}$

$\sum_{j=1}^{n} \frac{k_{j} \gamma_{j} P_{j}^{-\widetilde{\alpha}_{j}}}{\beta_{j}} \leq B$

As it is shown in Model (5), geometric mean is used for objective function defuzzification and the first constraint of Model (4) is defuzzified using strong comparison rule (SCR) method. The first, the second and the third constraints in (5) are not in posynomial form. To solve this problem we use a simple lemma from Beightler and Phillips (1976). To this end, consider the first constraint of the Model (5) that can be rewritten as follows:

$Z_{1}^{-1} k_{1}^{l} P_{1}^{\left(1-\alpha_{1}^{r}\right)}+Z_{1}^{-1} k_{2}^{l} P_{2}^{\left(1-\alpha_{2}^{r}\right)}+\cdots+Z_{1}^{-1} k_{n}^{l} P_{n}^{\left(1-\alpha_{n}^{r}\right)} \geq 1$

Parameter $t_{1 j}$ is defined as follow:

$t_{1 j}=\frac{k_{j}^{l} P_{j}^{\left(1-\alpha_{j}^{r}\right)}}{\sum_{j=1}^{n} k_{j}^{l} P_{j}^{\left(1-\alpha_{j}^{r}\right)}}, \quad j=1, \cdots, n$

where

$\sum_{j=1}^{n} t_{1 j}=1$

So the first constraint in (5) can be rewritten as follow:

$\prod_{j=1}^{n}\left(\frac{k_{j}^{l} P_{j}^{\left(1-\alpha_{j}^{r}\right)} Z_{l}^{-1}}{t_{1 j}}\right)^{-t_{1 j}} \leq 1$
Similarly, the define variables $t_{2 j}$ and $t_{3 j}$ as follow:

$$
\begin{aligned}
& t_{2 j}=\frac{k_{j}^{m} P_{j}^{\left(1-\alpha_{j}^{m}\right)}}{\sum_{j=1}^{n} k_{j}^{m} P_{j}^{\left(1-\alpha_{j}^{m}\right)}}, \quad j=1, \cdots, n \\
& t_{3 j}=\frac{k_{j}^{r} P_{j}^{\left(1-\alpha_{j}^{r}\right)}}{\sum_{j=1}^{n} k_{j}^{r} P_{j}^{\left(1-\alpha_{j}^{r}\right)}} . \quad j=1, \cdots, n
\end{aligned}
$$

As well as first constraint, the second and the third constraints can be reformulated as follows:

$\prod_{j=1}^{n}\left(\frac{k_{j}^{m} P_{j}^{\left(1-\alpha_{j}^{m}\right)} Z_{m}^{-1}}{t_{2 j}}\right)^{-t_{2 j}} \leq 1$,

$\prod_{j=1}^{n}\left(\frac{k_{j}^{r} P_{j}^{\left(1-\alpha_{j}^{r}\right)} Z_{r}^{-1}}{t_{3 j}}\right)^{-t_{3 j}} \leq 1$,

So, Model (5) can be rewritten as follow:

$\max \sqrt[3]{Z_{l} \cdot Z_{m} \cdot Z_{r}}$

subject to

$\prod_{j=1}^{n}\left(\frac{k_{j}^{l} P_{j}^{\left(1-\alpha_{j}^{r}\right)} Z_{l}^{-1}}{t_{1 j}}\right)^{-t_{1 j}} \leq 1$

$\prod_{j=1}^{n}\left(\frac{k_{j}^{m} P_{j}^{\left(1-\alpha_{j}^{m}\right)} Z_{m}^{-1}}{t_{2 j}}\right)^{-t_{2 j}} \leq 1$,

$\prod_{j=1}^{n}\left(\frac{k_{j}^{r} P_{j}^{\left(1-\alpha_{j}^{r}\right)} Z_{r}^{-1}}{t_{3 j}}\right)^{-t_{3 j}} \leq 1$,

$\sum_{j=1}^{n} \frac{k_{j}^{r} \gamma_{j} P_{j}^{-\alpha_{j}^{l}}}{\beta_{j}} \leq B$

To defuzzify fourth constraint of Model (5), SCR method is also used. Since the right hand side of the fourth constraint of Model (5) is crisp, defuzzification yields one constraint as fourth restriction of Model (13). 
Table 1. The results of the changes on different price elasticity items.

\begin{tabular}{lllllllll}
\hline & $\alpha$ & $p_{1}$ & $p_{2}$ & $p_{3}$ & $Z_{1}$ & $Z_{2}$ & $Z_{3}$ & $Z$ \\
\hline$\alpha 1+20 \%$ & $(4.56,5.04,5.4)$ & 5007 & 68030 & 118187 & 9860280 & 30225308 & 105743910 & 31586763 \\
$\alpha 1+20 \%$ & $(3.04,3.36,3.6)$ & 84652 & 68030 & 118187 & 9860280 & 30225308 & 105743910 & 31586763 \\
$\alpha 2+20 \%$ & $(3.24,3.36,3.48)$ & 19734 & 32688 & 57675 & 8119298 & 14976695 & 46267491.98 & 17785666 \\
$\alpha 2-20 \%$ & $(2.16,2.24,2.32)$ & 24898 & 981295 & 1893039 & 123363302 & 446238559 & 1570035471 & 442134273 \\
$\alpha 3+20 \%$ & $(2.52,2.64,2.7)$ & 23885 & 62173 & 117153 & 7818541 & 28266259 & 99467126 & 28012887 \\
$\alpha 3-20 \%$ & $(1.68,1.76,1.8)$ & 23020 & 448930 & 885278 & 125978910 & 230436379 & 708270551 & 273957183 \\
\hline
\end{tabular}

Note that Problem (8) is a parametric optimization problem. The regular procedure to solve (13) is to solve it different times. Each time, we estimate $t_{i j} ; i=1,2,3 ; j=1,2, \ldots, n$ using (7), (9) and (10) and the process continues until there are practically no changes on parameters. The experience shows that we need only a few iterations to reach the optimal solution.

The study presents the implementation of the developed model on one of ISPS located in IRAN that serves ADSL services.

\section{CASE STUDY}

The study implemented the proposed model of this paper for one of the ISPs located in Iran which offers ADSL services. The company offered three types of desired services: 128, 256, $512 \mathrm{~kb} / \mathrm{s}$.

Based on the experience of experts parameters $k_{\mathrm{j}} \mathrm{j}=1,2,3$ and $\alpha_{\mathrm{j}} j=1,2,3$ are estimated as follows:

$\alpha_{1}=[3.8,4.2,4.5]$,

$a_{2}=[2.7,2.8,2.9]$,

$a_{3}=[2.1,2.2,2.25]$

$k_{1}=\left[1.4 \times 10^{7}, 1.8 \times 10^{7}, 2.2 \times 10^{7}\right]$,

$\mathrm{k}_{2}=\left[1.0 \times 10^{7}, 1.2 \times 10^{7}, 1.4 \times 10^{7}\right]$,

$k_{3}=\left[7.2 \times 10^{12}, 7.6 \times 10^{12}, 7.8 \times 10^{12}\right]$

Other parameters are:

$\mathrm{b}_{1}=\mathrm{b}_{2}=\mathrm{b}_{3}=8$

$\mathrm{B}=51200 \mathrm{~kb} / \mathrm{sec}$

$\gamma_{1}=128 \mathrm{~kb} / \mathrm{sec}$

$\gamma_{2}=256 \mathrm{~kb} / \mathrm{sec}$

$\gamma_{3}=512 \mathrm{~kb} / \mathrm{sec}$

Problem is solved using CVX software and the optimal results are obtained as follow:

$$
\begin{aligned}
& P_{1}=2.3864 \times 10^{4}, P_{2}=6.8030 \times 10^{4}, P_{3}=1.1819 \times 10^{5} \\
& \tilde{Z}=\left(9.8603 \times 10^{6}, 3.0225 \times 10^{7}, 1.0574 \times 10^{6}\right)
\end{aligned}
$$

\section{Sensitivity analysis}

The changes on price elasticity of three products by decrease/increase in their values in a range of $20 \%$ were studied. Table 1 summarizes the experimental results. Also, the effects on changes in price elasticity on prices were studied. As can be observed from Table 1 the change on $\alpha_{1}$ only changes on the first service and it has no significant impact on the total revenue. Note that $k_{1}$ has relatively lower value compared with other ones and this is another reason to believe that any change on $\alpha_{1}$ does not change the overall revenue. In this case study, the change on the price of the first product does not make a big change on total revenue. On the other hand, based on the results of table, when the price of the first product increases the prices of the other two products remain with no changes. In fact, in some cases the price of the second service is higher than the first one. In such a case, customer may prefer to choose the second service due to higher internet speed and better availability. The increase on $\alpha_{2}$ has much more influence on prices since it has higher value of $k_{2}$. As can be observed from the table, a $20 \%$ change on the price of the second service reduces the price of the second and the third products, significantly. Since the second product is more popular among people where the case study is implemented, it can be concluded that this product has a higher competitive advantage against the other two products. The changes on the third product are not as significant as the impacts of price changes of the second product. In summary, it could be concluded that the second product plays an important role on the market.

\section{CONCLUSION}

In this paper, a mathematical model to formulate and solve pricing Internet Service Providers (ISP) problems 
was proposed. Developing model in the fuzzy environment increases model's stability against different real world situations. Whereas in the real world problems, most of effective parameters are estimated by experts as linguistic variables and on the other hand, linguistic terms usually quantified using fuzzy numbers, in the proposed model, uncertain parameters $k j ; j=1,2,3$ and $\alpha_{j} ; j=1,2,3$ are considered as fuzzy numbers with triangular possibility distribution. Finally, model is formulated as a possibilitic geometric programming and the effectiveness of the model has been illustrated via one of the ISP's located in Iran.

\section{REFRENCES}

Beightler CS, Phillips DT (1976). Applied Geometric Programming. Wiley, New York.

Belobaba P (1987b). Airline Yield Management: An Overview of Seat Inventory Control. Transport. Sci., 21(2): 63-73.

Bitran G, Caldentey R (2003). An overview of pricing models for revenue management. Int. J. Manufact. Serv. Oper. Manage., 5(3): .203-230

Burger B, Fuchs M (2005). Dynamic pricing - a future airline business model. Int. J. Revenue Pric. Manage., 4(1): 39-53.

Chiang W, Chen J, Xiaojing X (2007). An overview of research on revenue management: current issues and future research. Int. J. Revenue Manage., 1(1): 97-128.
Frank S (2003). Applying Six Sigma to revenue and pricing management. Int. J. Revenue Pric. Manage., 2(3): 245-254.

Kim D, Lee WJ (1998). Optimal joint pricing and lot sizing with fixed and variable capacity. Eur. J. Oper. Res., 109(1): 212-227.

Lee WJ (1993). Determining selling price and order quantity by geometric programming optimal solution, bounds and sensitivity. Decis. Sci., 24(1): 76-87.

McGill J, Van Ryzin G (1999). Revenue Management: Research Overview and prospects. Transport Sci., 33(2): 233-256.

Nair SK, Bapna P (2001). An application of yield management for Internet service Providers. Nav Res. Log., 48(5): 348-362

Sadjadi SJ , Oroujee M, Aryanezhad MB (2005). Optimal production and marketing planning . Comput Optim Appl., 30(2): 195-203.

Sadjadi SJ, Ghazanfari M, Yousefli A (2010). Fuzzy pricing and marketing planning model:a possibilistic geometric programming approach. Expert Syst. Appl., 37(4): 3392-3397. Omega Int. J. Manage., 37(2): 471-481. 